\title{
Liberación endoscópica del túnel cubital. Técnica y resultados clínico-funcionales
}

\author{
Endoscopic release of the ulnar tunnel. Technique \\ and clinical-functional outcomes
}

\author{
Solís-Villarruel O,* Sánchez-Gutiérrez LE
}

Hospital Regional de Alta Especialidad del Bajío.

RESUMEN. Introducción: El síndrome del túnel cubital es la segunda neuropatía por compresión de la extremidad superior, seguido del síndrome del túnel del carpo, por lo que es un motivo de consulta frecuente dentro de la cirugía de mano. Existen pocos estudios publicados acerca de la técnica endoscópica. Material y métodos: Se incluyeron 26 pacientes con el diagnóstico clínico y electromiográfico de síndrome de túnel cubital, en quienes se realizó liberación endoscópica del túnel cubital. Nueve (35\%) fueron mujeres y 17 (65\%) hombres. Se utilizó la clasificación de McGowan modificada y la clasificación de Wilson y Krout para analizar las características de las variables en su estado prequirúrgico y postquirúrgico. Resultados: Posterior a la intervención, obtuvimos 18 pacientes (69\%) con resultado excelente, seis (24\%) con un buen resultado y dos (7\%) con resultados regulares o aceptables, obteniendo una p significativa con un valor $<0.05$. Conclusión: La liberación endoscópica del túnel cubital se considera una técnica segura, con buenos resultados.

Palabras clave: Túnel cubital, nervio cubital, neuropatía, liberación endoscópica.

\section{Nivel de evidencia: IV}

\footnotetext{
* Postgrado en Cirugía de la Mano.

‡ Profesor Titular de Curso de Postgrado. Cirugía de la Mano.
}

Ortopedia y Traumatología. Hospital Regional de Alta Especiali`، d del Bajío.

Dirección para correspondencia:

Dr. Oliver Solís Villarruel

Global Médica: Calle Barrio de Tlaxcala Núm. 116,

Col. San Pedro, CP. 78178,

San Luis Potosí. Tel: 444-2549333.

E-mail: droliversolis@outlook.com

Este artículo puede ser consultado en versión completa en: www.medigraphic.com/actaortopedica
ABSTRACT. Introduction: The ulnar tunnel syndrome is the second compressive neuropathy, followed by the carpal tunnel, making it a frequent reference in hand surgery. There are few published studies about endoscopic technique. Material and methods: We studied 26 patients with ulnar tunnel syndrome diagnosis, were operated by endoscopic release of the ulnar tunnel. Nine women $(35 \%)$ and $17(65 \%)$ male patients. We used the modified McGowan, and the Wilson and Krout classification to analyze preoperative and postoperative variables. Results: After endoscopic decompression we obtained 18 patients (69\%) with excellent evolution, 6 (24\%) with good evolution, and $2(7 \%)$ with acceptable outcome. We obtained a significant $\mathrm{p}$ of $<0.05$. Conclusion: Endoscopic decompression of the ulnar tunnel is a safe technique, less invasive and with good outcome.

Keywords: Cubital tunnel, ulnar nerve, neuropathy, endoscopic decompression.

\section{Introducción}

La compresión del nervio cubital es la segunda neuropatía compresiva en frecuencia de la extremidad superior, detrás del síndrome del túnel carpiano., ${ }^{1,2}$ El nervio cubital puede estar comprimido en varios puntos a lo largo de su trayecto, pero la localización más frecuente es a nivel de codo, conocido como síndrome del túnel cubital. ${ }^{3,4}$ La posición superficial del nervio en el túnel cubital y el aumento de tensión y tracción que experimenta con la flexión del codo, lo hacen muy susceptible a esta neuropatía compresiva. ${ }^{5}$ La localización más frecuente de compresión es el túnel cubital, formado por el epicóndilo medial, el cúbito proximal, el límite 
tendinoso del flexor carpi ulnaris y la fascia entre éste y el ligamento arcuato de Osborne, mientras que a nivel proximal, la zona de mayor compresión es la arcada de Struthers, las localizaciones de compresión abarcan unos $8 \mathrm{~cm}$ proximales a $5 \mathrm{~cm}$ aproximadamente distales del epicóndilo medial. ${ }^{5,6}$

El motivo de consulta suelen ser parestesias en el territorio de distribución del nervio cubital. Pueden referir ocasionalmente dolor en la cara medial del codo que irradia el antebrazo, así como debilidad en la musculatura intrínseca. En casos severos y de larga evolución puede aparecer atrofia de la musculatura intrínseca, sobre todo en el primer músculo interóseo dorsal. ${ }^{7}$

Cuando falla el tratamiento conservador, se indica la liberación del nervio cubital a nivel del codo. Existen metaanálisis que han concluido que la descompresión simple tiene menos complicaciones que la transposición anterior. ${ }^{3,4,5,6,7,8}$ La liberación endoscópica es tan efectiva como la cirugía abierta, además es menos invasiva, preserva mejor la vascularidad del nervio y su recuperación es más rápida. ${ }^{2,3,4,5,6,7,8,9}$

El propósito de este estudio es observar los resultados clínicos y funcionales de pacientes con diagnóstico de síndrome de túnel cubital con la técnica de liberación endoscópica.

\section{Material y métodos}

En este estudio retroprolectivo se incluyeron 26 pacientes, a quienes se les realizó liberación endoscópica para el tratamiento del túnel cubital en el servicio de cirugía de la mano en un período de tres años. Se incluyeron los pacientes intervenidos con diagnóstico de síndrome del túnel cubital (basado en historia clínica, exploración física y estudio de neurofisiología) que cumplieran con los siguientes criterios: persistencia de clínica a pesar de tratamiento médico (terapia física, férula de limitación de flexión a $45^{\circ}$, fármacos, durante tres meses) y electromiografía con grado de moderado a severo. Se excluyeron aquéllos con patología cervical, patología ósea por radiografía, con intervención quirúrgica previa y/o antecedente de traumatismo.

Tabla 1: Clasificación de McGowan modificada de estatificación preoperatoria de la neuropatía cubital en el codo. ${ }^{5}$

$\begin{array}{cl}\text { Grado } & \text { Descripción } \\ \text { I } & \begin{array}{l}\text { Pacientes con síntomas subjetivos sensitivos, pero sin } \\ \text { hallazgos objetivos } \\ \text { Pacientes con buena fuerza de intrínsecos (4/5), sin } \\ \text { atrofia de intrínsecos }\end{array} \\ \text { IIA } & \begin{array}{l}\text { Pacientes con moderada fuerza de intrínsecos (3/5) } \\ \text { con atrofia } \\ \text { Pacientes con marcada atrofia de intrínsecos y altera- } \\ \text { ciones sensitivas }\end{array} \\ \text { IIB }\end{array}$

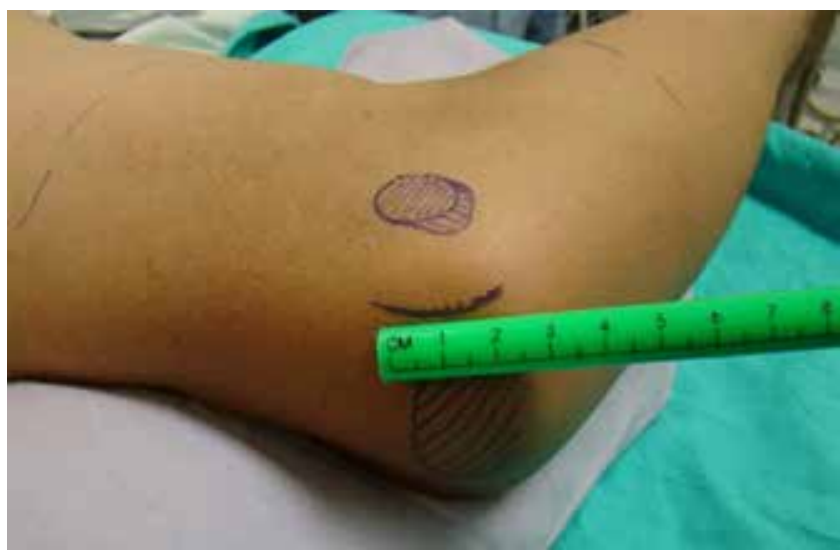

Figura 1: Incisión de $2 \mathrm{~cm}$ canal retroepitroclear.

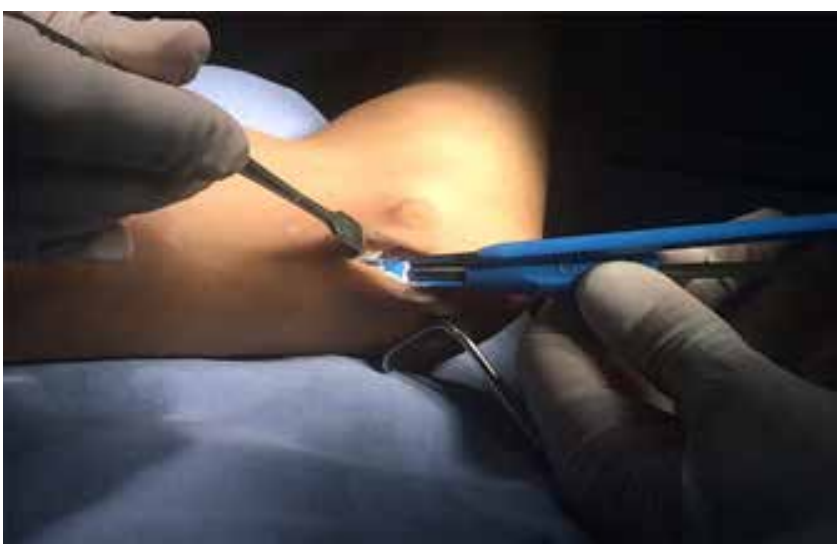

Figura 2: Liberación endoscópica proximal.

Del total de pacientes, nueve (35\%) fueron mujeres y 17 (65\%) hombres. Se clasificaron preoperatoriamente en cuatro grados mediante la clasificación modificada de McGowan (Tabla 1), ${ }^{5} 14$ pacientes (54\%) correspondían al grado 2A, 10 pacientes (38\%) al grado 2B y dos pacientes (8\%) al grado 3.

Empleamos la técnica de liberación endoscópica del túnel cubital descrita por Cobb. ${ }^{9,10}$ Mediante una incisión pequeña de $2 \mathrm{~cm}$, localizada a $1 \mathrm{~cm}$ retroepitroclear se localizó el nervio cubital (Figura 1), empleando los dilatadores y cánulas del sistema de liberación endoscópica de túnel del carpo de tipo desechable con ayuda de lente y fuente de luz de artroscopía $4.0 \mathrm{~mm}$ a 30 grados (Figuras 2 y 3 ).

Con la cuchilla del set de liberación se realizó la descompresión del nervio en ambas direcciones proximal y distal, debe avanzarse al mismo tiempo el lente artroscópico y la cuchilla a través de la cánula de liberación para que el nervio siempre quede protegido (Figura 4).

Previamente se ha comprobado, mediante visión endoscópica en el recorrido, que en los sitios a liberar no exista vaso ni rama nerviosa. A continuación, visualizamos el nervio liberado en todo su recorrido de los principales sitios de 


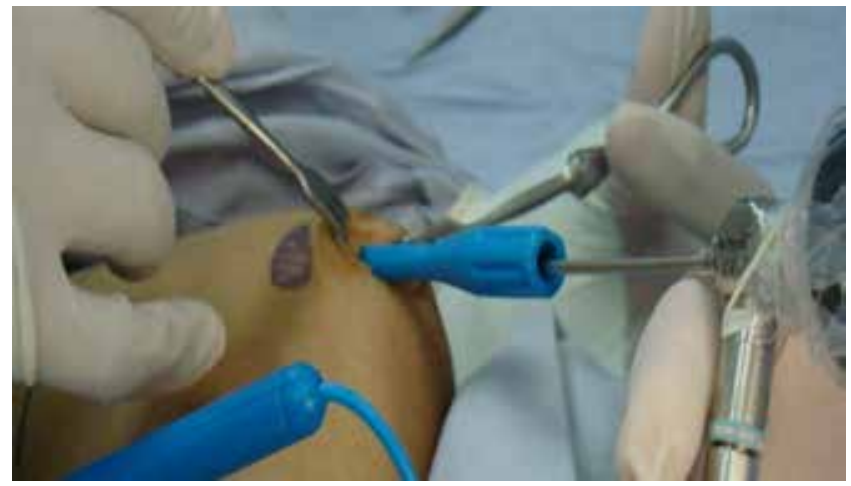

Figura 3: Liberación endoscópica distal.

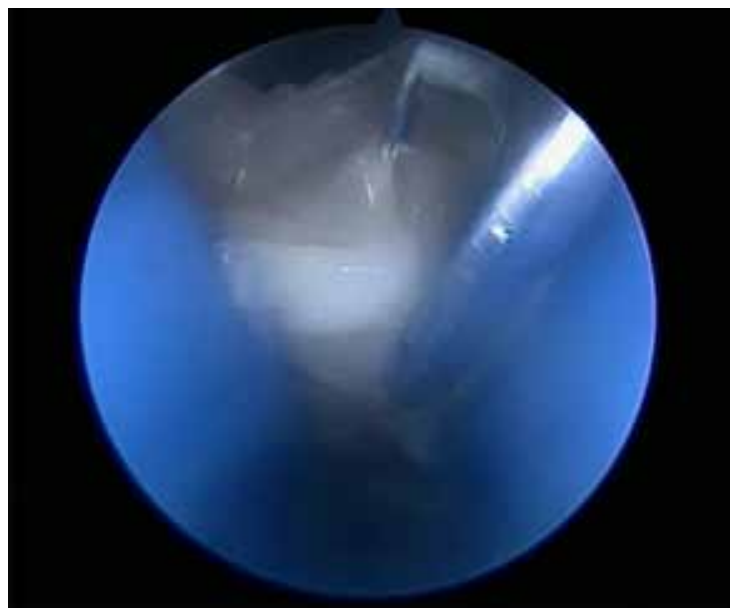

Figura 4: Vista endoscópica de liberación.

compresión $8 \mathrm{~cm}$ proximal y $5 \mathrm{~cm}$ distal de la epitróclea, abarcando desde la arcada de Struthers hasta el ligamento arcuato de Osborne (Figuras 5 y 6).

Los pacientes iniciaron movilización del codo al día siguiente de la intervención quirúrgica.

Se inició rehabilitación a los siete días. Se utilizó la escala de Wilson y Krout (Tabla 2$)^{5}$ para evaluación postquirúrgica. Los datos se analizaron con el paquete estadístico SPSS Statistics, versión 23, considerando una p significativa con un valor $<0.05$.

\section{Resultados}

Se incluyó un total de 26 pacientes, 17 (65\%) hombres y nueve (35\%) mujeres. La edad media fue de 44 años con rango de 29 a 54.

El codo izquierdo se vio afectado en 11 pacientes (42\%), mientras que el codo derecho en 15 (58\%).

Posterior a la liberación endoscópica del túnel cubital, 18 pacientes (69\%) presentaron un excelente resultado, seis pacientes (24\%) con un buen resultado y dos pacientes (7\%) con resultados aceptables, según la escala de Wilson y Krout (Tabla 2). En cuanto al tiempo de evolución de inicio de síntomas, nueve pacientes (34\%) contaban con menos de un año, 15 pacientes (58\%) con un año, y dos pacientes (8\%) tenían más de dos años de inicio de síntomas, estos últimos mostraron una evolución aceptable según la estadificación de Wilson y Krout.

Posterior a analizar estadísticamente la relación de la clasificación de McGowan con la clasificación de Wilson y Krout se obtuvo una relación significativa $(p=0.001)$ entre ambas variables.

El período de seguimiento de los pacientes fue de seis meses en consulta externa. No se registró ninguna complicación y/o recidiva postquirúrgica y todos los pacientes se reincorporaron a sus actividades.

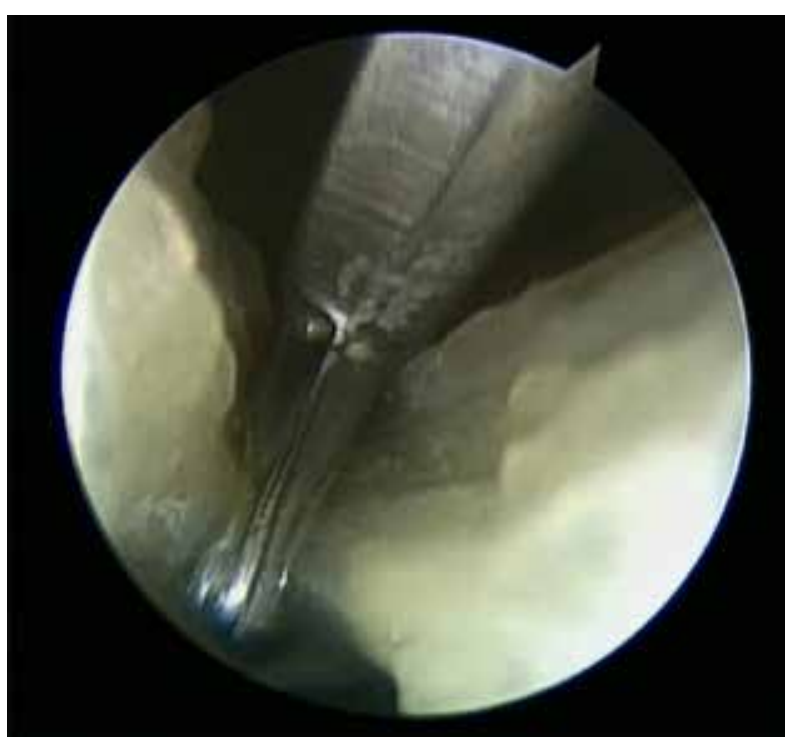

Figura 5: Liberación de arcada de Struthers.

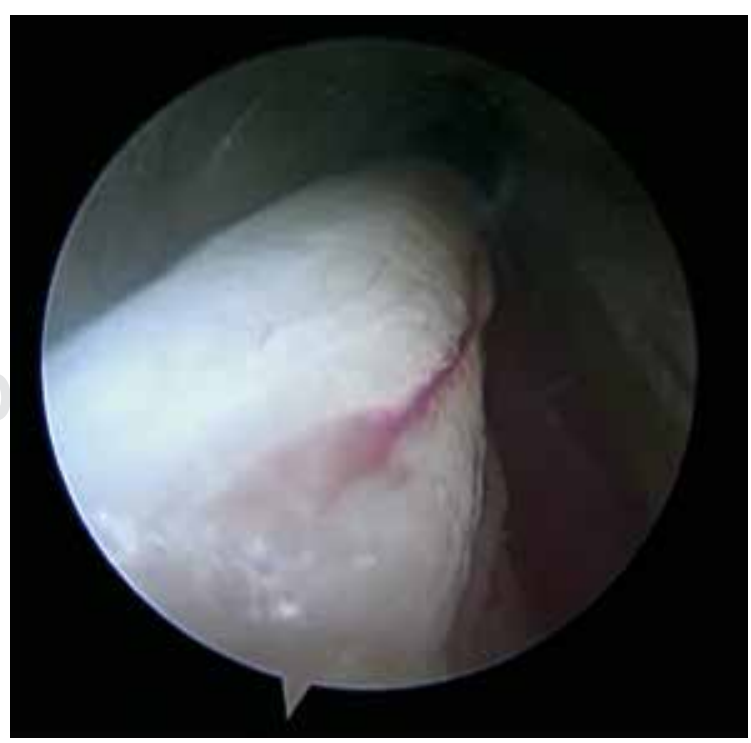

Figura 6: Nervio cubital liberado. 
Tabla 2: Clasificación de Wilson y Krout para la estadificación postoperatoria en pacientes con neuropatía cubital. ${ }^{5}$
Grado Descripción

Excelente Sin dolor en zona de la incisión, mínimas alteraciones sensitivas y motoras

Bueno Pérdida de los síntomas de la neuropatía, pero alteraciones sensitivas ocasionales

Regular Mejoría, pero persisten alteraciones sensitivas o motoras que son menores que antes de la intervención

Malo Sin mejoría o empeoramiento

\section{Discusión}

El atrapamiento del nervio cubital en el codo es una patología que cuenta con una amplia variedad de técnicas descritas para su tratamiento quirúrgico: descompresión simple o in situ, ya sea abierta o endoscópica, descompresión con transposición anterior del nervio, ya sea submuscular, intramuscular o subcutánea y anteriormente descompresión y epicondilectomía medial. Cada cirujano puede tener preferencia por una u otra, pero todavía hay un gran debate sobre cuál de las técnicas descritas es la mejor para el tratamiento quirúrgico de los pacientes con síndrome del túnel cubital. . $, 2,3,4,5,6,7,8$

Las técnicas de liberación endoscópica popularizadas por Bain y Bajhau, ${ }^{6}$ Tsai y colaboradores, ${ }^{11}$ Cobb $^{10}$ y Mirza y su equipo ${ }^{12}$ modificaron las técnicas endoscópicas existentes para túnel del carpo para ser utilizadas en el túnel cubital. Todas las técnicas permiten la liberación del nervio cubital a nivel de codo visualizando directamente el trayecto nervioso con mínimas incisiones. Desde que fue descrita por primera vez por Tsai y colegas ${ }^{13}$ han aparecido múltiples trabajos que han dado buenos resultados con la liberación endoscópica del túnel cubital y diversas modificaciones de la técnica original. ${ }^{11,12,13,14,15}$

En estudios que comparan la liberación endoscópica vs abierta, la descompresión endoscópica mostró menor índice de complicaciones y mejores resultados a corto plazo en términos de reincorporación laboral precoz, satisfacción del paciente y alivio del dolor. $2,3,4,5,6,7,7,8,9,10,11,12,13,14,15,16$

Con la liberación endoscópica del nervio cubital se pretende ofrecer varios beneficios al paciente en relación a la cirugía abierta, como minimizar el riesgo de lesión de estructuras adyacentes, reducir morbilidad del área quirúrgica, una recuperación más rápida y resultados estéticos más satisfactorios por el tamaño de la incisión. ${ }^{5,6,7,8,9,10,11,12,13,14,15,16,17}$ Además de lo anterior, las técnicas endoscópicas han demostrado ser seguras y eficaces, ${ }^{5,6,7,8,9,10,11,12,13,14,15,16,17,18}$ reportando buenos y excelentes resultados en alrededor de 70 a $80 \% .^{11,12,13,14,15,16,17}$

En nuestro estudio se utilizó la escala de Wilson y Krout modificada por Heithoff y colaboradores; además de síntomas neurológicos también analiza la clínica que se relaciona con la zona de incisión quirúrgica, siendo importante para el resultado final según Ahcan y Zorman. ${ }^{5,6,7,8,9,10,11,12,13,14,15,16,17,18,19}$

Obtuvimos $69 \%$ con excelente resultado, $24 \%$ con un buen resultado y $7 \%$ con resultados aceptables, sin complicaciones y/o recurrencia en los pacientes estudiados, lo que concuerda con otros autores en resultados favorables. ${ }^{4,5,6,7,7,8,9,10,11,12,13,14,15,16,17}$

$\mathrm{Al}$ igual que en otros estudios, se obtuvieron los mejores resultados en el grupo de pacientes con síntomas preoperatorios leves y moderados. 5,6,7,8,9,10,11,12,13,14,15,16,17 Sin embargo, podría aumentarse la muestra y evaluar en otro estudio tipo comparativo la elección de tratamiento y/o someterla a aleatorización.

Están aumentando los estudios comparativos del uso de técnicas de liberación del túnel cubital con una mínima incisión y todos los existentes muestran un beneficio. La limitación es el nivel de evidencia (tipo III), falta de grupos de control y tamaño de muestra relativamente pequeño.

\section{Conclusiones}

Con los resultados obtenidos, ésta es una técnica segura en el corto plazo, buena recuperación y resultados satisfactorios en la mayoría.

Bibliografía

1. Palmer BA, Hughes TB. Cubital tunnel syndrome. J Hand Surg Am. 2010; 35(1): 153-63.

2. Watts AC, Bain GI. Patient-rated outcomes of ulnar nerve decompression: a comparison of endoscopic and open in situ decompression. J Hand Surg Am. 2009; 34(8): 1492-8.

3. Zlowodzki M, Chan S, Bhandari M, Kalliainem L, Schubert W. Anterior transposition compared with simple descompression for treatment of cubital tunnel syndrome. A meta-analysis of randomized, controlled trials. J Bone Joint Surg Am. 2007; 89(12): 2591-8.

4. Gil GJ, Abellán GJ. Liberación endoscópica del túnel cubital. Técnica quirúrgica y resultados preliminares. Arch Med Dep. 2013; 30(6): 354-8.

5. Carratalá V, Lucas F, Sánchez AE, Calero R. Descompresión endoscópica del túnel cubital, técnica y experiencia. Rev Iberoam Cir Mano. 2014; 42(1): 9-17.

6. Bain GI, Bajhau A, Endoscopic release of the ulnar nerve at the elbow using the Agee device: a cadaveric study. Artrhoscopy. 2005; 21(6): 691-5.

7. Mitsionis GI, Manudis GN, Paschos NK. Comparative study of surgical treatment of ulnar nerve compression at the elbow. J Shoulder Elbow Surg. 2010; 19: 513-9.

8. Macadam SA, Gandhi R, Bezuhly M, Lefaivre KA. Simple descompression versus anterior subcutaneous and submuscular transposition of the ulnar nerve for cubital tunnel syndrome: a metaanalysis. J Hand Surg Am. 2008; 33(8): 1314-12.

9. Cobb T, Lemke J, Tyler J, Sterbank P. Efficacy of endoscopic cubital tunnel release. Hand. 2008; 3(2): 191.

10. Cobb TK, Endoscopic cubital tunnel release. J Hand Surg Am. 2010; 35(10): 1960-7.

11. Tsai TM, Chen IC, Majd ME, Lim BH. Cubital tunel release with endoscopic assistance: results of a new technique. J Hand Surg Am. 1999; 24(1): 21-9. 
12. Mirza A, Reinhart MK, Bove J, Litwa J. Scope-assisted release of the cubital tunnel. J Hand Surg Am. 2011; 36(1): 23-9.

13. Tsai TM, Bonczar M, Tsuruta T, Syed SA. A new operative technique: cubital tunnel decompression with endoscope assistance. Hand Clin. 1995; 11: 71-80.

14. Oertel J, Keiner D, Gaab MR. Endoscopic decompression of the ulnar nerve at the elbow. Neurosurgery. 2010; 66(4): 817-24.

15. Jiang S, Xu W, Shen Y, Xu JG, Gu YD. Endoscopy-assited cubital tunnel release under carbon dioxide insufflations and anterior transposition. Ann Plast Surg. 2012; 68(1): 62-6.
16. Dützmann S, Martin KD, Sobottka S, Marquardt G, Schackert G, Seifert V, et al. Open vs retractor-endoscopic in situ decompression of the ulnar nerve in cubital tunnel syndrome a retrospective cohort study. Neurosurgery. 2013; 72(4): 605-16.

17. Hoffmann R, Siemionow M. The endoscopic management of cubital tunnel syndrome. J Hand Surg Br. 2006; 31(1): 23-9.

18. Nakao Y, Takayama S, Toyama Y. Cubital tunnel release with lift-type endoscopic surgery. Hand Surg. 2001; 6: 199-203.

19. Achan U, Zorman P. Endoscopic decompression of the ulnar nerve at the elbow. J Hand Surg Am. 2007; 32(8): 1171-6. 